\title{
Global Attractivity and Oscillations in a Nonlinear Impulsive Parabolic Equation with Delay
}

\author{
XiaO WANG \\ Department of Mathematics and System Science, College of Science, National Uni- \\ versity of Defense Technology, Changsha, 410073, P. R. China \\ e-mail: wxiao_98@yahoo.com.cn

\section{ZHIXIANG Li} \\ Department of Mathematics and System Science, College of Science, National Uni- \\ versity of Defense Technology, Changsha, 410073, P. R. China \\ e-mail : zhxli02@yahoo.com.cn
}

ABstract. Global attractivity and oscillatory behavior of the following nonlinear impulsive parabolic differential equation which is a general form of many population models

$$
\left\{\begin{array}{l}
\frac{\partial u(t, x)}{\partial t}=\Delta u(t, x)-\delta u(t, x)+f(u(t-\tau, x)), t \neq t_{k} \\
u\left(t_{k}^{+}, x\right)-u\left(t_{k}, x\right)=g_{k}\left(u\left(t_{k}, x\right)\right), k \in I_{\infty}
\end{array}\right.
$$

are considered. Some new sufficient conditions for global attractivity and oscillation of the solutions of $(*)$ with Neumann boundary condition are established. These results not only are true but also improve and complement existing results for $(*)$ without diffusion or impulses. Moreover, when these results are applied to the Nicholson's blowflies model and the model of Hematopoiesis, some new results are obtained.

\section{Introduction}

There has been increasing interest in impulsive partial differential system [1], $[2],[5],[6],[7],[8],[9],[12],[13]$ since the first paper [4] was published which has shown that impulsive partial differential equations provided a natural framework for mathematical modeling of population growth in 1991. As far as we know, the authors in [4], [8], [9], [18] have studied the global asymptotic stability of unique positive equilibrium for this kind of systems, however, there are almost nobody to consider this fields with delay. Furthermore, although the oscillations for the kind of systems were studied in many papers [1], [2], [5], [6], [7], [8], [9], [12], [13], the nonlinear terms $f(u)$ and $g_{k}(u)$ were assumed to be odd and $f(u)$ was also provided to be convex in most of these papers. In fact, many models for population growth need to be described by a class of nonlinear impulsive partial differential system with delay in which the nonlinear term $f(u)$ and $g_{k}(u)$ are not needed to be odd

Received July 25, 2006.

2000 Mathematics Subject Classification: 35R12, 35R10.

Key words and phrases: impulsive parabolic equation, global attractivity, oscillation. 
or convex. For example, Nicholson's blowflies model and model of Hematopoiesis. Obviously, it is meaningful to investigate behavior of solution of the kind of systems.

In this paper, motivated by Erbe, Freedman, Liu and Wu [4], Gao and Wang [8], Redlinger [15], [16] and Yang and So [20], using the solutions of impulsive ordinary differential equations with delay, we first define a pair of lower-upper solution of a class of nonlinear impulsive parabolic system with delay. Then, the main results on the global attractivity are established by applying the method of lower-upper solution pair for this system and a comparison theorem proved by us. Our oscillation result is obtained by using an oscillation theory which is developed by us and parallels to the one in Kulenovic, Ladas and Meimaridou [11] and Yang and So [20] for delay differential equations.

We note that our main results in this paper are also valid when this nonlinear parabolic system is not effected by impulses or a spatial variable $x \in \Omega$.

The rest of this paper is organized as follows. In Section 2, we give some notations and definitions. Section 3 is devoted to establish and prove some preliminary information needed in the following. In section 4, the attractivity of positive equilibrium is considered, while the oscillatory behavior of solutions about the positive equilibrium is considered in section 5 . In section 6 , we use these results in diffusive Nicholson's blowflies model with impulse and diffusive model of Hematopoiesis with impulse and obtain several new results.

\section{Notations and definitions}

In this paper, we consider the following nonlinear impulsive differential system with delay:

$$
\left\{\begin{array}{l}
\frac{\partial u(t, x)}{\partial t}=\Delta u(t, x)-\delta u(t, x)+f(u(t-\tau, x)), t \neq t_{k}, t>0 \\
\frac{\partial u(t, x)}{\partial \nu}=0, t \neq t_{k},(t, x) \in \Gamma \equiv[0, \infty) \times \partial \Omega \\
u\left(t_{k}^{+}, x\right)-u\left(t_{k}, x\right)=g_{k}\left(u\left(t_{k}, x\right)\right), k \in I_{\infty},(t, x) \in D \equiv[0, \infty) \times \Omega
\end{array}\right.
$$

with initial condition

$$
u(\theta, x)=\phi(\theta, x) \geq 0, \quad(\theta, x) \in D_{\tau} \equiv[-\tau, 0] \times \bar{\Omega},
$$

where $\delta>0, \tau>0, I_{\infty}=\{1,2, \cdots\} ; \Omega \subset R^{n}$ is a bounded domain with a smooth boundary $\partial \Omega ; \Delta u(t, x)=\sum_{i=1}^{n} \frac{\partial^{2} u(t, x)}{\partial x_{i}^{2}} ; \frac{\partial}{\partial \nu}$ denotes the exterior normal derivative on $\partial \Omega ; 0<t_{1}<t_{2}<\cdots<t_{k}<\cdots, \inf _{i \in N}\left\{t_{i+1}-t_{i}\right\}=\gamma>0$ and $\lim _{k \rightarrow \infty} t_{k}=\infty$.

Throughout this paper, we always assume that there exist three positive constants $y_{0}>0, K>0, M_{0}>0$ such that

(H1) $f(y) \in C^{1}\left(R, R^{+}\right), f(y)$ is increasing on $\left(0, y_{0}\right)$ and decreasing on $\left(y_{0}, \infty\right)$, $f\left(y_{0}\right)=\max _{y \in[0, \infty)} f(y), f(0)=0$.

(H2) $f(y)>\delta y$ for any $y \in(0, K)$ and $f(y)<\delta y$, for any $y \in(K, \infty)$. 
(H3) $g_{k} \in C^{1}\left(R^{+}, R\right)$ for any $k \in I_{\infty}, x+g_{k}(x)$ is increasing for $x \in R_{+}$and satisfies

$$
\begin{gathered}
x \geq x+g_{k}(x) \geq K, x \geq K, \\
x \leq x+g_{k}(x)<K, 0<x<K, \\
\left|g_{k}(x)\right| \leq M_{0}, x \geq 0 .
\end{gathered}
$$

It is very important to investigate behavior of solution of system (2.1)-(2.2). In fact, (2.1) can describe many models for population growth, among which are the diffusive Nicholson's blowflies model with impulse:

$$
\left\{\begin{array}{l}
\frac{\partial u(t, x)}{\partial t}=\Delta u(t, x)-\delta u(t, x)+p u(t-\tau, x) e^{-a u(t-\tau, x)}, t \neq t_{k}, t>0 \\
\frac{\partial u(t, x)}{\partial \nu}=0, t \neq t_{k},(t, x) \in \Gamma \\
u\left(t_{k}^{+}, x\right)-u\left(t_{k}, x\right)=g_{k}\left(u\left(t_{k}, x\right)\right), k \in I_{\infty},(t, x) \in D
\end{array}\right.
$$

and the diffusive model of Hematopoiesis with impulse:

$$
\left\{\begin{array}{l}
\frac{\partial u(t, x)}{\partial t}=\Delta u(t, x)-r u(t, x)+\frac{\beta u(t-\tau, x)}{1+u^{m}(t-\tau, x)}, t \neq t_{k}, t>0 \\
\frac{\partial u(t, x)}{\partial \nu}=0, t \neq t_{k},(t, x) \in \Gamma \\
u\left(t_{k}^{+}, x\right)-u\left(t_{k}, x\right)=g_{k}\left(u\left(t_{k}, x\right)\right), k \in I_{\infty},(t, x) \in D
\end{array}\right.
$$

When $g_{k}(x) \equiv 0,(2.3)$ has been studied by Yang and So [20] and when $g_{k}(x) \equiv 0$ and $u(t, x) \equiv u(t),(2.4)$ was first proposed by Mackey and Glass[14] to describe some physiological control system and has been investigated by Kuang [10] and Saker[17].

For convenience, we introduce the following notations as that in Erbe et.al [4]

$$
\begin{gathered}
P_{k}=\left\{\left(t_{k}, x\right) ; x \in \Omega\right\}, P=\cup_{k=1}^{\infty} P_{k} ; \\
\Lambda_{k}=\left\{\left(t_{k}, x\right) ; x \in \partial \Omega\right\}, \Lambda=\cup_{k=1}^{\infty} \Lambda_{k}
\end{gathered}
$$

and define $P C^{1,2}\left(D_{\tau} \cup \bar{D}, P\right)$ as the set of all functions $u(t, x): D_{\tau} \cup \bar{D} \rightarrow R$ satisfying the following conditions:

(i) $u(t, x)$ is continuously differentiable for $(t, x) \in D_{\tau} \cup \bar{D} \backslash(P \cup \Lambda)$;

(ii) $u_{x x}(t, x)$ exists and is continuous for $(t, x) \in D \backslash P$;

(iii) for $v=\left(u, u_{t}, u_{x}, u_{x x}\right), \lim _{(s, y) \rightarrow_{s<t_{k}}\left(t_{k}, x\right)} v(s, y)=v\left(t_{k}, x\right)$ and $\lim _{(s, y) \rightarrow s>t_{k}}\left(t_{k}, x\right) v(s, y)=v\left(t_{k}^{+}, x\right)$ exist for $k \in I_{\infty}$ and $x \in \bar{\Omega}$, where $u_{x}=$ $\left(\frac{\partial u}{\partial x_{1}}, \cdots, \frac{\partial u}{\partial x_{n}}\right), u_{x x}=\left(\frac{\partial^{2} u}{\partial x_{1}^{2}}, \frac{\partial^{2} u}{\partial x_{1} x_{2}} \cdots, \frac{\partial^{2} u}{\partial x_{n}^{2}}\right)$ and $u_{t}=\frac{\partial u}{\partial t}$.

Definition 2.1. A function $u(t, x) \in P C^{1,2}\left(D_{\tau} \cup \bar{D}, P\right)$ is said to be a solution of the initial boundary value problem (IBVP for short) (2.1)-(2.2) if it satisfies (2.1)$(2.2)$. 
Definition 2.2. A non-zero solution $u(t, x)$ of system (2.1) is said to be nonoscillatory in $D$ if it is either eventually positive or eventually negative. Otherwise, it is called oscillatory in $D$.

\section{Some lemmas and corresponding proofs}

In order to prove our main results, we first give and prove some lemmas. Consider the following three impulsive differential equations with delay:

$$
\begin{aligned}
& \left\{\begin{array}{l}
\frac{\partial u(t, x)}{\partial t}=\Delta u(t, x)-\delta u(t, x)+F(u(t-\tau, x)), \quad(t, x) \in D \backslash P, \\
\frac{\partial u(t, x)}{\partial \nu}=0,(t, x) \in \Gamma \backslash \Lambda, \\
u(\theta, x)=\phi(\theta, x) \geq 0,(\theta, x) \in D_{\tau}, \\
u\left(t_{k}^{+}, x\right)-u\left(t_{k}, x\right)=G_{k}\left(u\left(t_{k}, x\right)\right), x \in \bar{\Omega}, k \in I_{\infty},
\end{array}\right. \\
& \left\{\begin{array}{l}
w^{\prime}(t)=-\delta w(t)+F^{1}(w(t-\tau)) \\
w(\theta)=\max _{(\theta, x) \in D_{\tau}} \phi(\theta, x) \\
w\left(t_{k}^{+}\right)=w\left(t_{k}\right)+G_{k}^{1}\left(w\left(t_{k}\right)\right)
\end{array}\right.
\end{aligned}
$$

and

$$
\left\{\begin{array}{l}
v^{\prime}(t)=-\delta v(t)+F^{2}(v(t-\tau)) \\
v(\theta)=\min _{(\theta, x) \in D_{\tau}} \phi(\theta, x) \\
v\left(t_{k}^{+}\right)=v\left(t_{k}\right)+G_{k}^{2}\left(v\left(t_{k}\right)\right)
\end{array}\right.
$$

We assume that

(C1) $G_{k}, G_{k}^{1}, G_{k}^{2} \in C(R, R)$, for any $x, y, z \in R, x \leq y \leq z$, satisfy $x+G_{k}^{2}(x) \leq$ $y+G_{k}(y) \leq z+G_{k}^{1}(z)$.

(C2) $F, F^{1}, F^{2} \in C(R, R)$, for any $x, y, z \in R, x \leq y \leq z$, satisfy $F^{2}(x) \leq$ $F(y) \leq F^{1}(z)$.

Lemma 3.1. Assume that the conditions $(\mathrm{C} 1)$ and $(\mathrm{C} 2)$ hold, $w(t)$ and $v(t)$ are the solutions of (3.2) and (3.3), then every solution of (3.1) satisfies $v(t) \leq u(t, x) \leq$ $w(t), \quad(t, x) \in \bar{D}$.

Proof. For any $T>0$, let $Q_{T}=[-\tau, T] \times \bar{\Omega}$, and there is a positive integer $n$ such that $t_{n} \leq T<t_{n+1}$. We first prove that $v(t) \leq u(t, x) \leq w(t), \quad(t, x) \in Q_{T}$.

For any $\varepsilon>0$, let $w(t, \varepsilon)$ and $v(t, \varepsilon)$ be solutions of the two following equations on $Q_{T}$

and

$$
\left\{\begin{array}{l}
w^{\prime}(t, \varepsilon)=-\delta w(t, \varepsilon)+F^{1}(w(t-\tau, \varepsilon))+\varepsilon \\
w(\theta, \varepsilon)=\max _{(\theta, x) \in D_{\tau} \phi(\theta, x)+\varepsilon} \\
w\left(t_{k}^{+}, \varepsilon\right)=w\left(t_{k}, \varepsilon\right)+G_{k}^{1}\left(w\left(t_{k}, \varepsilon\right)\right)+\varepsilon
\end{array}\right.
$$

$$
\left\{\begin{array}{l}
v^{\prime}(t, \varepsilon)=-\delta v(t, \varepsilon)+F^{2}(v(t-\tau, \varepsilon))-\varepsilon \\
v(\theta, \varepsilon)=\min _{(\theta, x) \in D_{\tau}} \phi(\theta, x)-\varepsilon \\
v\left(t_{k}^{+}, \varepsilon\right)=v\left(t_{k}, \varepsilon\right)+G_{k}^{2}\left(v\left(t_{k}, \varepsilon\right)\right)-\varepsilon
\end{array}\right.
$$


It is not difficult to verify that $\lim _{\varepsilon \rightarrow 0} v(t, \varepsilon)=v(t), \lim _{\varepsilon \rightarrow 0} w(t, \varepsilon)=w(t)$ uniformly on $[-\tau, T]$. We claim that

$$
v(t, \varepsilon)<u(t, x)<w(t, \varepsilon), \quad(t, x) \in Q_{T} .
$$

Since the proof of the case $u(t, x)<w(t, \varepsilon),(t, x) \in Q_{T}$ is the same as the case $v(t, \varepsilon)<u(t, x),(t, x) \in Q_{T}$, we just prove that $v(t, \varepsilon)<u(t, x),(t, x) \in Q_{T}$. Let $m_{\varepsilon}(t, x)=u(t, x)-v(t, \varepsilon)$. If the conclusion is not true, then there exists $t_{0}>0$ and $x_{0} \in \bar{\Omega}$ such that one of the following three cases holds:

Case $1 \quad t_{0} \neq t_{k}, 1 \leq k \leq n, m_{\varepsilon}\left(t_{0}, x_{0}\right)=0$ and $m_{\varepsilon}(t, x)>0$ on $\left(0, t_{0}\right) \times \bar{\Omega}$;

Case $2 t_{0}=t_{k}$, for some $k, 1 \leq k \leq n, m_{\varepsilon}\left(t_{k}, x_{0}\right)=0$ and $m_{\varepsilon}(t, x)>0$ on $\left(0, t_{k}\right) \times \bar{\Omega}$;

Case $3 t_{0}=t_{k}$, for some $k, 1 \leq k \leq n, m_{\varepsilon}\left(t_{k}^{+}, x_{0}\right) \leq 0$ and $m_{\varepsilon}(t, x)>0$ on $\left(0, t_{k}\right] \times \bar{\Omega}$.

In Case 1 , if $\left(t_{0}, x_{0}\right) \in(0, T] \times \partial \Omega$. For any $(t, x) \in\left[0, t_{0}\right] \times \bar{\Omega}$, we have

$$
\begin{aligned}
& \frac{\partial m_{\varepsilon}}{\partial t}-\Delta m_{\varepsilon}(t, x)+\delta m_{\varepsilon}(t, x) \\
= & \frac{\partial u}{\partial t}-\Delta u-v^{\prime}(t, \varepsilon)+\delta(u(t, x)-v(t, \varepsilon)) \\
= & F(u(t-\tau, x))-F^{2}(v(t-\tau, \varepsilon))+\varepsilon \\
\geq & \varepsilon>0 .
\end{aligned}
$$

Note $\delta>0$ and by strong minimum principle of Hopf, we have

$$
\frac{\partial u\left(t_{0}, x_{0}\right)}{\partial \nu}=\frac{\partial m_{\varepsilon}\left(t_{0}, x_{0}\right)}{\partial \nu}<0
$$

which leads to a contradiction with $\frac{\partial u\left(t_{0}, x_{0}\right)}{\partial \nu}=0$. Hence $\left(t_{0}, x_{0}\right) \in(0, T] \times \Omega$. Since $m_{\varepsilon}(t, x)$ attains its minimum at $\left(t_{0}, x_{0}\right)$, we have

$$
\frac{\partial m_{\varepsilon}\left(t_{0}, x_{0}\right)}{\partial t} \leq 0 \text { and } \triangle m_{\varepsilon}\left(t_{0}, x_{0}\right) \geq 0 .
$$

Note the condition $(\mathrm{C} 2),(3.1),(3.4)$ and the second inequality of (3.5), we get

$$
\begin{aligned}
& \frac{\partial m_{\varepsilon}\left(t_{0}, x_{0}\right)}{\partial t} \\
= & \Delta m_{\varepsilon}\left(t_{0}, x_{0}\right)-\delta m_{\varepsilon}\left(t_{0}, x_{0}\right)+F\left(u\left(t_{0}-\tau, x_{0}\right)\right)-F^{2}\left(v\left(t_{0}-\tau, \varepsilon\right)\right)+\varepsilon \\
\geq & \Delta m_{\varepsilon}\left(t_{0}, x_{0}\right)+\varepsilon \\
\geq & \varepsilon>0,
\end{aligned}
$$

which is a contradiction with the first inequality of (3.5).

By using the same type of argument as in the proof Case 1 one obtains that Case 2 is not true. 
In Case 3 , note that $m_{\varepsilon}\left(t_{k}, x_{0}\right)>0$, we obtain

$$
\begin{aligned}
& 0 \geq m_{\varepsilon}\left(t_{k}^{+}, x_{0}\right) \\
= & m_{\varepsilon}\left(t_{k}, x_{0}\right)+G_{k}\left(u\left(t_{k}, x_{0}\right)\right)-G_{k}^{2}\left(v\left(t_{k}, \varepsilon\right)\right)+\varepsilon \\
\geq & \varepsilon>0
\end{aligned}
$$

which leads to a contradiction.

Therefore, we have $v(t, \varepsilon)<u(t, x),(t, x) \in Q_{T}$, and

$$
v(t)=\lim _{\varepsilon \rightarrow 0} v(t, \varepsilon) \leq u(t, x),(t, x) \in Q_{T} .
$$

Similarly, we get

$$
w(t) \geq u(t, x),(t, x) \in Q_{T} .
$$

Since $T$ is arbitrary we have

$$
v(t) \leq u(t, x) \leq w(t),(t, x) \in[-\tau, \infty) \times \bar{\Omega} .
$$

The proof of Lemma 3.1 is complete.

Remark 3.1. Assume that $m=\min _{(t, x) \in[-\tau, \infty) \times \bar{\Omega}} u(t, x), M=\min _{(t, x) \in[-\tau, \infty) \times \bar{\Omega}} u(t, x)$. Lemma 3.1 is also valid if the assumption (C2) is replaced by the following weaker condition

(C3) $F, F^{1}, F^{2} \in C(R, R)$, for any $x, y, z \in[m, M], x \leq y \leq z$, satisfy $F^{2}(x) \leq$ $F(y) \leq F^{1}(z)$.

Consider the two following impulsive differential inequalities,

$$
\left\{\begin{array}{l}
w^{\prime}(t) \geq-\delta w(t)+F^{1}(w(t-\tau)) \\
w(\theta) \geq \max _{(\theta, x) \in D_{\tau}} \phi(\theta, x) \\
w\left(t_{k}^{+}\right) \geq w\left(t_{k}\right)+G_{k}^{1}\left(w\left(t_{k}\right)\right)
\end{array}\right.
$$

and

$$
\left\{\begin{array}{l}
v^{\prime}(t) \leq-\delta v(t)+F^{2}(v(t-\tau)), \\
v(\theta) \leq \min _{(\theta, x) \in D_{\tau}} \phi(\theta, x) \\
v\left(t_{k}^{+}\right) \leq v\left(t_{k}\right)+G_{k}^{2}\left(v\left(t_{k}\right)\right)
\end{array}\right.
$$

Definition 3.1. $(v, w)$ is said to be a lower-upper solution pair of (3.1) if the conditions (C1) and (C3) hold and $v(t)$ and $w(t)$ are solutions of (3.6) and (3.7) respectively.

Remark 3.2. From Lemma 3.1, it is not difficult to see that if $(v, w)$ is a lowerupper solution pair of (3.1) then every solution of (3.1) satisfies $v(t) \leq u(t, x) \leq$ $w(t),(t, x) \in[-\tau, \infty) \times \bar{\Omega}$. 
Assume that $q>0, \tau>0$ and $b_{k}>-1, k \in I_{\infty}$. Consider the three following impulsive differential systems:

$$
\begin{gathered}
\left\{\begin{array}{l}
\frac{\partial u(t, x)}{\partial t}-\Delta u(t, x)+q u(t-\tau, x)=0, t \neq t_{k}, \\
\frac{\partial u(t, x)}{\partial \nu}=0, \\
u\left(t_{k}^{+}, x\right)-u\left(t_{k}, x\right)=b_{k} u\left(t_{k}, x\right), k \in I_{\infty},(t, x) \in D,
\end{array}\right. \\
\left\{\begin{array}{l}
U^{\prime}(t)+q U(t-\tau)=0, t \neq t_{k}, \\
U\left(t_{k}^{+}\right)-U\left(t_{k}\right)=b_{k} U\left(t_{k}\right), k \in I_{\infty},
\end{array}\right.
\end{gathered}
$$

and

$$
y^{\prime}(t)+q \prod_{t-\tau \leq t_{k}<t}\left(1+b_{k}\right)^{-1} y(t-\tau)=0 .
$$

Lemma 3.2(Li et al. [13]). Every solution of system (3.8) oscillates in $D$ if and only if every solution of system (3.9) oscillates.

Lemma 3.3(Yan et al. [19]). Every solution of system (3.9) oscillates if and only if every solution of system (3.10) oscillates.

By Lemma 3.2 and Lemma 3.3, we can easily get the following Lemma.

Lemma 3.4. Every solution of system (3.8) oscillates in $D$ if and only if every solution of system (3.10) oscillates.

It is known from the work of Yan et al. [19] and Lemma 3.4, one can easily get the following result.

Lemma 3.5. If

$$
q \liminf _{t \rightarrow \infty} \int_{t-\tau}^{t} \prod_{s-\tau \leq t_{k}<s}\left(1+b_{k}\right)^{-1} d s>\frac{1}{e} .
$$

then every solution of system (3.8) oscillates in D.

Now we consider the relationship between system (3.8) and the following impulsive differential system

$$
\left\{\begin{array}{l}
\frac{\partial u(t, x)}{\partial t}=\Delta u(t, x)-Q(t, x) u(t-\tau, x), t \neq t_{k}, \\
\frac{\partial u(t, x)}{\partial \nu}=0, \\
u\left(t_{k}^{+}, x\right)-u\left(t_{k}, x\right)=h_{k}\left(t_{k}, x\right) u\left(t_{k}, x\right), k \in I_{\infty},(t, x) \in D,
\end{array}\right.
$$


where $h_{k} \in C\left(R \times R^{n},(-1, \infty)\right)$, for any $k \in I_{\infty}$ such that

$$
\lim _{t \rightarrow \infty} h_{k}(t, x)=b_{k}>-1 \text {, uniformly in } x
$$

and $Q(t, x) \in C\left(R \times R^{n}, R^{+}\right)$satisfies

$$
\lim _{t \rightarrow \infty} Q(t, x)=q>0 \text {, uniformly in } x .
$$

Lemma 3.6. If (3.11), (3.13) and (3.14) hold then every solution of system (3.12) oscillates in $D$.

Proof. If (3.11) holds, then one can take a $\varepsilon>0$ such that $0<\varepsilon \ll q$ and

$$
(q-\varepsilon) \liminf _{t \rightarrow \infty} \int_{t-\tau}^{t} \prod_{s-\tau \leq t_{k}<s}\left(1+b_{k}+\varepsilon\right)^{-1} d s>\frac{1}{e} .
$$

In fact, for any $q>\varepsilon>0$, let

$$
\eta(\varepsilon)=(q-\varepsilon) \liminf _{t \rightarrow \infty} \int_{t-\tau}^{t} \prod_{s-\tau \leq t_{k}<s}\left(1+b_{k}+\varepsilon\right)^{-1} d s
$$

It follows from (3.11) that $\eta(\varepsilon)$ is continuous and decreasing on $[0, \infty), \eta(\varepsilon) \leq$ $\eta(0)=\eta$. If $\eta=\infty$, then we let $\frac{q}{2}>\varepsilon>0$ be small enough such that $\eta(\varepsilon) \geq \frac{\eta}{2}$. In this case, (3.15) is true; if $0<\eta<\infty,(3.15)$ is also valid by continuity of $\eta(\varepsilon)$.

Suppose for the purpose of contradiction that (3.12) has an eventually positive solution $u(t, x)$. Then there exists a sufficiently large $T_{1}>0$ such that $u(t, x)>0$ and $u(t-\tau, x)>0,(t, x) \in\left[T_{1}, \infty\right) \times \bar{\Omega}$. Let $\varepsilon>0$ be sufficiently small such that (3.15) and by (3.13), (3.14), there exists a sufficiently large $T>T_{1}$ such that

$$
-\varepsilon<h_{k}(t, x)-b_{k}<\varepsilon, k \in I_{\infty},
$$

and

$$
-\varepsilon<Q(t, x)-q<\varepsilon .
$$

for any $(t, x) \in[T, \infty) \times \bar{\Omega}$. Hence, by (3.12), (3.16) and (3.17), we have $u(t, x)$ is an eventually positive solution of the following inequality:

$$
\left\{\begin{array}{l}
\frac{\partial u(t, x)}{\partial t}-\Delta u(t, x)+(q-\varepsilon) u(t-\tau, x) \leq 0, t \neq t_{k}, \\
\frac{\partial u(t, x)}{\partial \nu}=0, \\
u\left(t_{k}^{+}, x\right)-u\left(t_{k}, x\right) \leq\left(b_{k}+\varepsilon\right) u\left(t_{k}, x\right), k \in I_{\infty},(t, x) \in D,
\end{array}\right.
$$

However, from Lemma 3.4, Lemma 3.5 and (3.15), one can get that (3.18) hasn't any eventually positive solution, which is a contradiction. 
Similarly, (3.12) hasn't any eventually negative solution. The proof of Lemma 3.6 is complete.

Consider the impulsive differential system

$$
\left\{\begin{array}{l}
\frac{\partial u(t, x)}{\partial t}=\Delta u(t, x)-\delta u(t, x)-Q(t, x) u(t-\tau, x), t \neq t_{k} \\
\frac{\partial u(t, x)}{\partial \nu}=0 \\
u\left(t_{k}^{+}, x\right)-u\left(t_{k}, x\right)=h_{k}\left(t_{k}, x\right) u\left(t_{k}, x\right), k \in I_{\infty},(t, x) \in D .
\end{array}\right.
$$

Let $u(t, x)=e^{-\delta t} v(t, x)$, then

$$
\left\{\begin{array}{l}
\frac{\partial v(t, x)}{\partial t}=\Delta v(t, x)-e^{\delta \tau} Q(t, x) v(t-\tau, x), t \neq t_{k} \\
\frac{\partial u(t, x)}{\partial \nu}=0 \\
v\left(t_{k}^{+}, x\right)-v\left(t_{k}, x\right)=h_{k}\left(t_{k}, x\right) v\left(t_{k}, x\right), k \in I_{\infty},(t, x) \in D .
\end{array}\right.
$$

Since the change of variable $u(t, x)=e^{-\delta t} v(t, x)$ does not change the oscillation of solution, we have

Corollary 3.7. Assume that (3.13), (3.14) and $e^{\delta \tau} q \lim \inf _{t \rightarrow \infty} \int_{t-\tau}^{t} \prod_{s-\tau \leq t_{k}<s}(1+$ $\left.b_{k}\right)^{-1} d s>\frac{1}{e}$ hold then every solution of (3.19) oscillates in D.

Remark 3.3. When $u(t, x)$ does not depend a spatial variable $x \in \Omega$ or $h_{k}(t, x) \equiv 0$ for any $k \in I_{\infty}$, the corresponding results are also true.

\section{Global attractivity of the positive equilibrium}

In this section, we will prove the global attractivity of the positive equilibrium $K$ when $K<y_{0}$. We first prove the following theorem.

Theorem 4.1. If (H1)-(H3)hold, then the following statements are true:

(i) every solution $u(t, x)$ of (2.1)-(2.2) satisfies

$$
0 \leq \limsup _{t \rightarrow \infty} u(t, x) \leq \frac{f\left(y_{0}\right)}{\delta}+\frac{M_{0}}{1-e^{\delta \gamma}} \text {, uniformly in } x .
$$

(ii) every solution $u(t, x)$ of $(2.1)-(2.2)$ satisfies $u(t, x) \geq 0,(t, x) \in(0, \infty) \times \bar{\Omega}$.

(iii) if $\phi(\theta, x) \not \equiv 0,(t, x) \in D_{\tau}$, then every solution $u(t, x)$ of (2.1)-(2.2) satisfies $u(t, x)>0,(t, x) \in(\tau, \infty) \times \bar{\Omega}$.

Proof. (i) Let $w(t)$ be the solution of the following equation

$$
\left\{\begin{array}{l}
w^{\prime}(t)=-\delta w(t)+f\left(y_{0}\right), t \geq 0, t \neq t_{k} \\
w(0)=\max _{(t, x) \in D_{\tau}} \phi(t, x), t \in[-\tau, 0] \\
w\left(t_{k}^{+}\right)=w\left(t_{k}\right)+g_{k}\left(w\left(t_{k}\right)\right), k \in I_{\infty}
\end{array}\right.
$$


It follows from (H1)-(H3) and Definition 3.1 that $(0, w)$ is a lower-upper pair of (2.1)-(2.2). So by Remark 3.2, we have

$$
0 \leq u(t, x) \leq w(t),(t, x) \in[-\tau, \infty) \times \bar{\Omega} .
$$

Note that the solution $w(t)$ of (4.1) can be written as

$$
w(t)=\frac{f\left(y_{0}\right)}{\delta}+e^{-\delta t}\left(w(0)-\frac{f\left(y_{0}\right)}{\delta}\right)+\sum_{0<t_{k}<t} e^{-\delta\left(t-t_{k}\right)} g_{k}\left(w\left(t_{k}\right)\right), t \geq 0 .
$$

Let $t_{k}<t<t_{k+1}$, by $\inf _{i \in N}\left\{t_{i+1}-t_{i}\right\}=\gamma>0$, we have

$$
t-t_{1}>t_{k}-t_{1}=\sum_{i=1}^{k-1}\left(t_{i+1}-t_{i}\right) \geq(k-1) \gamma .
$$

Therefore,

$$
\sum_{0<t_{k}<t} e^{-\delta\left(t-t_{k}\right)} \leq \sum_{i=0}^{\infty} e^{-\delta \gamma i}=\frac{1}{1-e^{\delta \gamma}}
$$

According to the above formula, (4.2),(4.3) and (H3), we get

$$
0 \leq \limsup _{t \rightarrow \infty} u(t, x) \leq \limsup _{t \rightarrow \infty} w(t) \leq \frac{f\left(y_{0}\right)}{\delta}+\frac{M_{0}}{1-e^{\delta \gamma}} .
$$

(ii) By (4.2),(ii) is valid.

(iii) In view of (ii), we have $u(t, x) \geq 0,(t, x) \in \bar{D}$. There are now two possible cases to consider.

Case 1, $\phi(0, x) \not \equiv 0$. We claim that $u(t, x)>0,(t, x) \in(0, \infty) \times \bar{\Omega}$. If the conclusion is not true, then there exists $\left(t_{0}, x_{0}\right) \in(0, \infty) \times \bar{\Omega}$ such that one of the following three subcases holds:

Subcases 1.1 , for any $k \geq 1, t_{0} \neq t_{k}, u(t, x)>0$ on $\left(0, t_{0}\right) \times \bar{\Omega}$ and $u\left(t_{0}, x_{0}\right)=0$. If $t_{0} \neq t_{k},\left(t_{0}, x_{0}\right) \in \partial \Omega$, then by strong minimum principle of Hopf, we have $\frac{\partial u\left(t_{0}, x_{0}\right)}{\partial \nu}<0$, which leads to a contradiction with $\frac{\partial u\left(t_{0}, x_{0}\right)}{\partial \nu}=0$. Hence $\left(t_{0}, x_{0}\right) \in$ $(0, \infty) \times \Omega$, but it is impossible according to the minimum principle.

Subcases $1.2, t_{0}=t_{k}$ for some $k \in I_{\infty}, u(t, x)>0$ on $\left(0, t_{k}\right) \times \bar{\Omega}$ and $u\left(t_{k}, x_{0}\right)=$ 0 . By using the same type of argument as in the proof of Subcase 1.1 one obtains that Subcases 1.2 is not true.

Subcases $1.3, t_{0}=t_{k}$ for some $k \in I_{\infty}, u(t, x)>0$ on $\left(0, t_{k}\right] \times \bar{\Omega}$ and $u\left(t_{k}^{+}, x_{0}\right) \leq 0$. If $u\left(t_{k}^{+}, x_{0}\right)=0$, then by (H3), we have $u\left(t_{k}, x_{0}\right)=0$, which is a contradiction with $u\left(t_{k}, x_{0}\right)>0$; if $u\left(t_{k}^{+}, x_{0}\right)<0$, then it follows from (H3), we have $0>u\left(t_{k}^{+}, x_{0}\right)=$ $u\left(t_{k}, x_{0}\right)+g_{k}\left(u\left(t_{k}, x_{0}\right)\right) \geq 0$, which leads a contradiction.

Case 2, $\phi(0, x) \equiv 0, x \in \bar{\Omega}$. We first show that $u(t, x) \not \equiv 0$ for $(t, x) \in(0, \tau] \times \bar{\Omega}$. Suppose not. From $(2.1)$, we have $\phi(\theta, x) \equiv 0$ for $(\theta, x) \in D_{\tau}$, which contradicts the assumption $\phi(\theta, x) \not \equiv 0$ on $D_{\tau}$. Therefore there exists $t_{0} \in(0, \tau]$ such that 
$u\left(t_{0}, x\right) \not \equiv 0$ for $x \in \bar{\Omega}$. Now using the same argument as in Case 1 one can show that $u(t, x)>0$ for $(t, x) \in\left(t_{0}, \infty\right) \times \bar{\Omega}$. The proof of Theorem 4.1 is complete.

Theorem 4.2. Assume that (H1)-(H3) and $M_{0} \leq\left(y_{0}-\frac{f\left(y_{0}\right)}{\delta}\right)\left(1-e^{-\delta \gamma}\right)$. If $K<y_{0}$, then every solution $u(t, x)$ of (2.1)-(2.2) satisfies $\lim _{t \rightarrow \infty} u(t, x)=$ $K$, uniformly in $x$.

Proof. In view of Theorem 4.1(i) and $M_{0} \leq\left(y_{0}-\frac{f\left(y_{0}\right)}{\delta}\right)\left(1-e^{-\delta \gamma}\right)$, we may assume without loss of generality that every solution $u(t, x)$ of (2.1)-(2.2) satisfies

$$
0 \leq u(t, x) \leq \frac{f\left(y_{0}\right)}{\delta}+\frac{M_{0}}{1-e^{\delta \gamma}} \leq y_{0}, \quad \text { on } \quad D_{\tau} \cup \bar{D} .
$$

Let $\underline{U}(t)=\min _{x \in \bar{\Omega}} u(t, x), \bar{U}(t)=\max _{x \in \bar{\Omega}} u(t, x), \underline{U}=\liminf _{t \rightarrow \infty} \underline{U}(t)$ and $\bar{U}=$ $\lim \sup _{t \rightarrow \infty} \bar{U}(t)$. By (4.4), we have

$$
0 \leq \underline{U} \leq \bar{U} \leq y_{0}
$$

From Theorem 4.1(iii), set

$$
z_{0}=\min \left\{\min _{(t, x) \in[2 \tau, \infty) \times \bar{\Omega}} u(t, x), \frac{K}{2}\right\}>0 .
$$

Now we define two sequences $\left\{z_{n}\right\}$ and $\left\{y_{n}\right\}$ to satisfy, respectively,

$$
z_{n}=\frac{f\left(z_{n-1}\right)}{\delta}, n \in I_{\infty},
$$

and

$$
y_{n}=\frac{f\left(y_{n-1}\right)}{\delta}, n \in I_{\infty} .
$$

We prove that $\left\{z_{n}\right\}$ and $\left\{y_{n}\right\}$ are monotonic and bounded. First of all, we prove $\left\{z_{n}\right\}$ is monotonically increasing and $K$ is the least upper bounded. Note that $f(y)>\delta y$, for any $y \in(0, K), z_{0}<K$ and $f(y)$ is increasing on $\left[z_{0}, K\right] \subset\left[0, y_{0}\right]$ we have

$$
z_{1}=\frac{f\left(z_{0}\right)}{\delta}>z_{0}, \quad \text { and } \quad z_{1}=\frac{f\left(z_{0}\right)}{\delta}<\frac{f(K)}{\delta}=K .
$$

By induction and direct computation, we have

$$
0<z_{0}<z_{1}<\cdots<\lim _{n \rightarrow \infty} z_{n}=K .
$$

Similarly, we have

$$
0>y_{0}>y_{1}>\cdots>\lim _{n \rightarrow \infty} y_{n}=K .
$$


Without loss of generality, we assume that $t_{1}>3 \tau$ and define $v_{1}(t)$ and $w_{1}(t)$ to be the solution of the following differential equation, respectively,

$$
\left\{\begin{array}{l}
v_{1}^{\prime}(t)=-\delta\left[v_{1}(t)-z_{1}\right], t \geq 3 \tau, t \neq t_{k} \\
v_{1}(\theta)=z_{0}<K, \theta \in[2 \tau, 3 \tau] \\
v_{1}\left(t_{k}^{+}\right)=v_{1}\left(t_{k}\right)+g_{k}\left(v_{1}\left(t_{k}\right)\right)
\end{array}\right.
$$

and

$$
\left\{\begin{array}{l}
w_{1}^{\prime}(t)=-\delta\left[w_{1}(t)-y_{1}\right], t \geq 3 \tau, t \neq t_{k} \\
w_{1}(\theta)=y_{0}>K, \theta \in[2 \tau, 3 \tau] \\
w_{1}\left(t_{k}^{+}\right)=w_{1}\left(t_{k}\right)+g_{k}\left(w_{1}\left(t_{k}\right)\right)
\end{array}\right.
$$

Therefore, from (H1), (H3) and Definition 3.1, it is not difficult to verify that $\left(v_{1}(t), w_{1}(t)\right)$ is a lower-upper pair of $(2.1)$ with initial condition $z_{0} \leq u(t, x) \leq y_{0}$ on $[2 \tau, 3 \tau] \times \bar{\Omega}$. Consequently, by Remark 3.2 , we have

$$
v_{1}(t) \leq u(t, x) \leq \omega_{1}(t) \quad \text { on } \quad[2 \tau, \infty) \times \bar{\Omega} .
$$

For $t \in\left[3 \tau, t_{1}\right]$, by (4.7) and (4.9), we have

$$
v_{1}(t)=z_{1}+e^{-\delta(t-3 \tau)}\left(z_{0}-z_{1}\right) \geq z_{0},
$$

and

$$
z_{0} \leq v_{1}\left(t_{1}\right)=z_{1}+e^{-\delta\left(t_{1}-3 \tau\right)}\left(z_{0}-z_{1}\right) \leq z_{1}<K .
$$

Moreover, by (H3) we have

$$
z_{0} \leq v_{1}\left(t_{1}\right) \leq v_{1}\left(t_{1}^{+}\right)=v_{1}\left(t_{1}\right)+g_{1}\left(v_{1}\left(t_{1}\right)\right)<K .
$$

For $t \in\left(t_{1}, t_{2}\right]$, from $(4.7),(4.9)$ and $(4.12)$, we get

$$
v_{1}(t)=z_{1}+e^{-\delta\left(t-t_{1}\right)}\left(v_{1}\left(t_{1}^{+}\right)-z_{1}\right) \geq z_{0},
$$

and

$$
v_{1}\left(t_{2}\right)=z_{1}+e^{-\delta\left(t_{2}-t_{1}\right)}\left(v_{1}\left(t_{1}^{+}\right)-z_{1}\right) \leq z_{1}\left(1-e^{-\delta\left(t_{2}-t_{1}\right)}\right)+e^{-\delta\left(t_{2}-t_{1}\right)} K<K .
$$

Also, by (H3) we obtain

$$
z_{0} \leq v_{1}\left(t_{2}\right) \leq v_{1}\left(t_{2}^{+}\right)=v_{1}\left(t_{1}\right)+g_{2}\left(v_{1}\left(t_{2}\right)\right)<K \text {. }
$$

Applying this argument repeatedly, one can easily show that $v_{1}(t)$ is increasing on $[3 \tau, \infty)$ and such that

$$
v_{1}(t)=z_{1}+e^{-\delta(t-3 \tau)}\left(z_{0}-z_{1}\right)+\sum_{3 \tau<t_{k}<t} e^{-\delta\left(t-t_{k}\right)} g_{k}\left(v_{1}\left(t_{k}\right)\right), t \geq 3 \tau .
$$


Therefore, we get

$$
z_{1} \leq \lim _{t \rightarrow \infty} v_{1}(t)
$$

Similarly, by using the same argument as the above, one can show that the solution $w_{1}(t)$ of $(4.10)$ is decreasing on $[3 \tau, \infty)$ and such that

$$
\lim _{t \rightarrow \infty} w_{1}(t) \leq y_{1}
$$

Note that $v_{1}(t)$ and $w_{1}(t)$ are monotonic functions and (4.11), (4.13) and (4.14), we have

$$
z_{1} \leq \underline{U} \leq \bar{U} \leq y_{1}
$$

Define $v_{n}(t)$ and $w_{n}(t)$ to be the solutions of the following differential equations, respectively,

$$
\left\{\begin{array}{l}
v_{n}^{\prime}(t)=-\delta\left[v_{n}(t)-z_{n}\right], t \geq 3 \tau, t \neq t_{k}, \\
v_{n}(\theta)=z_{n-1}<K, \theta \in[2 \tau, 3 \tau] \\
v_{n}\left(t_{k}^{+}\right)=v_{n}\left(t_{k}\right)+g_{k}\left(v_{n}\left(t_{k}\right)\right)
\end{array}\right.
$$

and

$$
\left\{\begin{array}{l}
w_{n}^{\prime}(t)=-\delta\left[w_{n}(t)-y_{n}\right], t \geq 3 \tau, t \neq t_{k}, \\
w_{n}(\theta)=y_{n-1}>K, \theta \in[2 \tau, 3 \tau], \\
w_{n}\left(t_{k}^{+}\right)=w_{n}\left(t_{k}\right)+g_{k}\left(w_{n}\left(t_{k}\right)\right) .
\end{array}\right.
$$

Repeating the above procedure, we have the following relation,

$$
z_{0}<z_{1}<\cdots<z_{n} \leq \underline{U} \leq \bar{U} \leq y_{n}<\cdots<y_{1}<y_{0} .
$$

By (4.7), (4.8) and (4.15), we have

$$
K=\lim _{n \rightarrow \infty} z_{n} \leq \underline{U} \leq \bar{U} \leq \lim _{n \rightarrow \infty} y_{n}=K
$$

which implies

$$
\lim _{t \rightarrow \infty} u(t, x)=K, \quad \text { uniformly in } x .
$$

The proof of Theorem 4.2 is complete.

If $g_{k}(x) \equiv 0$ for any $k \in I_{\infty}$ in $(2.1)$, then from the proof of Theorem 4.2 , one easily obtains the following result.

Corollary 4.3. Suppose that (H1) and (H2)hold. If $K \leq y_{0}$, then every solution $u(t, x)$ of (2.1)-(2.2) satisfies $\lim _{t \rightarrow \infty} u(t, x)=K$, uniformly in $x$.

\section{Oscillation about the positive equilibrium}

In this section we will show that under some additional restrictions on the time delay $\tau, f^{\prime}(K)$ and $g_{k}^{\prime}(K)$ for any $k \in I_{\infty}$, all non-trivial solutions of (2.1)-(2.2) oscillate about positive equilibrium $K$ given $K>y_{0}$. Now, we give and prove our 
main result of this section.

Theorem 5.1. Assume that (H1)-(H3) hold. If $y_{0}\left\langle K, g_{k}^{\prime}(K)\right\rangle-1$ for any $k \in I_{\infty}$ and

$$
-e^{\delta \tau} f^{\prime}(K) \liminf _{t \rightarrow \infty} \int_{t-\tau}^{t} \prod_{s-\tau \leq t_{k}<s}\left(1+g_{k}^{\prime}(K)\right)^{-1} d s>\frac{1}{e},
$$

then every solution $u(t, x)$ of (2.1)-(2.2) oscillates about positive equilibrium $K$ in $D$.

Proof. Let $u(t, x)=v(t, x)+K$, then (2.1)-(2.2) can be rewritten as follows:

$$
\left\{\begin{array}{l}
\frac{\partial v(t, x)}{\partial t}=\Delta v(t, x)-\delta v(t, x)+f(v(t-\tau, x)+K)-\delta K, t \neq t_{k}, t>0, \\
\frac{\partial v(t, x)}{\partial \nu}=0, t \neq t_{k},(t, x) \in \Gamma, \\
v\left(t_{k}^{+}, x\right)-v\left(t_{k}, x\right)=g_{k}\left(v\left(t_{k}, x\right)+K\right), k \in I_{\infty}, x \in \Omega,
\end{array}\right.
$$

and

$$
v(\theta, x)=\phi(\theta, x)-K \geq-K, \quad(\theta, x) \in D_{\tau} .
$$

Clearly, it follows from Definition 2.2, the non-trivial solution $u(t, x)$ of $(2.1)-(2.2)$ oscillates about positive equilibrium $K$ if and only if $v(t, x)$ of $(5.2)-(5.3)$ oscillates about zero. So we will only show that $v(t, x)$ of $(5.2)-(5.3)$ oscillates about zero. If the conclusion is not valid, then (5.2)-(5.3) has an eventually positive solution $v(t, x)$ or eventually negative solution $v(t, x)$. We will show that either eventually positive solution $v(t, x)$ or eventually negative solution $v(t, x)$ satisfies

$$
\lim _{t \rightarrow \infty} v(t, x)=0, \text { uniformly in } x .
$$

By Theorem 4.1, it is not difficult to verify that $v(t, x)$ is bounded. Now consider $v(t, x)$, two cases are possible:

Case $1, v(t, x)$ is a eventually positive solution. That is, there exists $T>0$ such that

$$
v(t, x)>0, v(t-\tau, x)>0, \forall(t, x) \in[T, \infty) \times \bar{\Omega} .
$$

Note that $v(t-\tau, x)+K>K$ for $(t, x) \in[T, \infty) \times \bar{\Omega}$ and assumptions (H1)-(H3) and $y_{0}<K$,we have

$$
\left\{\begin{array}{l}
\frac{\partial v(t, x)}{\partial t} \leq \Delta v(t, x)-\delta v(t, x), t \neq t_{k}, t>0 \\
\frac{\partial v(t, x)}{\partial \nu}=0, t \neq t_{k},(t, x) \in \Gamma \\
v\left(t_{k}^{+}, x\right)-v\left(t_{k}, x\right) \leq 0, k \in I_{\infty}, x \in \Omega
\end{array}\right.
$$

By using the same type argument as in the proof Lemma 3.1 and (5.5) we can get that (5.4) is correct. 
Case 2, $v(t, x)$ is a eventually negative solution. Without loss of generality we assume that $-K<v(t, x)<0$, for all $t \in[-\tau, \infty) \times \bar{\Omega}$. Let

$$
g(y)=f(y+K)-\delta K=f(y+K)-f(K) .
$$

Since $f$ is increasing on $\left[0, y_{0}\right]$ and decreasing on $\left[y_{0}, \infty\right)$ and $y_{0}<K, g(y)$ is increasing on $\left[-K, y_{0}-K\right]$ and decreasing on $\left[y_{0}-K, 0\right]$. Hence, from $g(-K)=$ $-\delta K<0$ and $g\left(y_{0}-K\right)=f\left(y_{0}\right)-f(K)>0$, there exists a unique zero point $A$ of $g(y)$ for $-K<y<0$. Let

$$
G(y)= \begin{cases}g(y), & \text { for } y \in[-K, A] \\ 0, & \text { for } y \in(A, 0]\end{cases}
$$

Consider the delay differential equation

$$
\left\{\begin{array}{l}
w^{\prime}(t)=-\delta w(t)+G(w(t-\tau)), t \geq 0 \\
w(\theta)=\min _{x \in \bar{\Omega}} v(\theta, x)<0, \theta \in[-\tau, 0] .
\end{array}\right.
$$

It is not difficult to verify that $\delta y \leq G(y)$ for $-K<y<0$. Hence, there exists a constant $\alpha \in(-K, 0]$ such that $\lim _{t \rightarrow \infty} w(t)=\alpha(\operatorname{Ding}[3]$ Theorem 2). We claim that $\alpha=0$. In fact if $\alpha \in(-K, A]$, then by the first formula of (5.6),we have

$$
\lim _{t \rightarrow \infty} v^{\prime}(t)=-\delta(\alpha+K)+f(\alpha+K)>0,
$$

which is a contradiction. Hence $\alpha \in(A, 0)$. Similarly, by (5.6), we get

$$
\lim _{t \rightarrow \infty} v^{\prime}(t)=-\delta \alpha>0
$$

which is a contradiction. Therefore,

$$
\lim _{t \rightarrow \infty} w(t)=0 .
$$

If we are able to prove that $w(t) \leq v(t, x)<0$ for all $(t, x) \in[0, \infty) \times \bar{\Omega}$, then (5.4) is true. In view of lemma 3.1, to prove $w(t) \leq v(t, x)<0$ for all $(t, x) \in[0, \infty) \times \bar{\Omega}$, it suffices to show that

$$
g(u) \geq G(v), \quad \text { for any } u, v \in[-K, 0] \text { and } u \geq v .
$$

There are three cases to consider:(i) $A \geq u \geq v$, (ii) $u \geq A \geq v$, (iii) $u \geq v \geq A$.

In case (i), (5.7) is true because $G(y)=g(y)$ and $g(y)$ is increasing for $y \in$ $[-K, A]$. In case (ii), (5.7) is also correct since $G(v)=g(v) \leq 0$, while, $g(u) \geq 0$. In case (iii), (5.7) again holds since $G(v)=0$ and $g(u) \geq 0$.

Therefore, summarizing the above discussion, (5.4) is valid.

Let $h_{k}\left(t_{k}, x\right)=\frac{g_{k}\left(v\left(t_{k}, x\right)+K\right)}{v\left(t_{k}\right)}, Q(t, x)=\frac{f(v(t, x)+K)-\delta K}{v(t, x)}$. Then,

can be rewritten as

$$
\left\{\begin{array}{l}
\frac{\partial v(t, x)}{\partial t}=\Delta v(t, x)-\delta v(t, x)+Q(t, x) v(t, x), t \neq t_{k}, t>0 \\
\frac{\partial v(t, x)}{\partial \nu}=0, t \neq t_{k},(t, x) \in \Gamma \\
v\left(t_{k}^{+}, x\right)-v\left(t_{k}, x\right)=h_{k}\left(t_{k}, x\right) v\left(t_{k}, x\right), k \in I_{\infty}, x \in \Omega
\end{array}\right.
$$


By (5.4), we have

$$
\lim _{t \rightarrow \infty} h_{k}(t, x)=g_{k}^{\prime}(K)>-1, \text { for any } k \in I_{\infty}, \text { uniformly in } x
$$

and

$$
\lim _{t \rightarrow \infty} Q(t, x)=f^{\prime}(K), \text { uniformly in } x .
$$

However, Corollary 3.1 and (5.1) imply that every solution of (5.2)-(5.3) oscillates in $D$, which is a contradiction. The proof of Theorem 5.1 is complete.

If $g_{k}(x) \equiv 0$ for any $k \in I_{\infty}$ in (2.1),then from Corollary 4.1 and the proof of Theorem 5.1, the following results are easily obtained.

Corollary 5.2. If (H1) and (H2) hold then every non-oscillatory solution $u(t, x)$ of (2.1)-(2.2) about positive equilibrium $K$ in $D$ satisfies $\lim _{t \rightarrow \infty} u(t, x)=$ $K$, uniformly in $x$.

Corollary 5.3. Assume that (H1) and (H2) hold. In addition, if $y_{0}<K$ and $-\tau e^{\delta \tau} f^{\prime}(K)>\frac{1}{e}$, then every solution $u(t, x)$ of $(2.1)-(2.2)$ oscillates about positive equilibrium $K$ in $D$.

\section{Applications}

We illustrate our main results to (2.3) and (2.4) in this section. First of all, for (2.3), we assume that $\delta, \tau, p$ and $a$ are positive constants. Suppose that $\frac{p}{\delta}>1$. Take $y_{0}=\frac{1}{a}, K=\frac{1}{a} \ln \frac{p}{\delta}$ and $f(y)=p y e^{-a y}$. It is not difficult to see that the function $f(y)$ satisfies assumptions (H1) and (H2). Therefore, applying the above results to (2.3), we have the following statements.

(i) If $1<\frac{p}{\delta}<e$ holds, then $K=\frac{1}{a} \ln \frac{p}{\delta}<\frac{1}{a}=y_{0}$. Hence if $g_{k}(x)$ satisfies (H3) and $M_{0} \leq \frac{1}{a}\left(1-\frac{p}{\delta e}\right)\left(1-e^{-\delta \gamma}\right)$, from Theorem 4.2, then every solution $u(t, x)$ of (2.3) under condition (2.2) converges to $K$ (uniformly in $\mathrm{x}$ )as $t \rightarrow \infty$.

(ii) If $\frac{p}{\delta}>e$ holds, then $K=\frac{1}{a} \ln \frac{p}{\delta}>\frac{1}{a}=y_{0}$. Therefore, by Theorem 5.1, if $g_{k}(x)$ satisfies $(\mathrm{H} 3)$ and $g_{k}^{\prime}(K)>-1$ and $e^{\delta \tau} \delta\left(\ln \frac{p}{\delta}-1\right) \liminf _{t \rightarrow \infty} \int_{t-\tau}^{t} \prod_{s-\tau \leq t_{k}<s}(1+$ $\left.g_{k}^{\prime}(K)\right)^{-1} d s>\frac{1}{e}$, then every solution $u(t, x)$ of (2.3) under condition (2.2) oscillates about positive equilibrium $K$ in $D$.

If $g_{k}(x) \equiv 0$ for any $k \in I_{\infty}$ in (2.3), then we have the following results.

(iii) If $1<\frac{p}{\delta} \leq e$ holds, then from Corollary 4.3, every solution $u(t, x)$ of $(2.3)$ under condition (2.2) converges to $K$ (uniformly in x)as $t \rightarrow \infty$.

(iv) If $\frac{p}{\delta}>e$ and $-\tau e^{\delta \tau} f^{\prime}(K)=\tau e^{\delta \tau} \delta\left(\ln \frac{p}{\delta}-1\right)>\frac{1}{e}$ hold, then from Corollary 
5.3 , every solution $u(t, x)$ of (2.3) under condition (2.2) oscillates about positive equilibrium $K$ in $D$.

(v) If $\frac{p}{\delta}>1$, then by Corollary 5.2, every non-oscillatory solution $u(t, x)$ of $(2.3)$ under condition (2.2) about positive equilibrium $K$ in $D$ converges to $K$ (uniformly in $\mathrm{x}$ )as $t \rightarrow \infty$.

Remark 6.1. When $\frac{p}{\delta}>1$, (v) complements the results in Yang and So [20]. Moreover, (iii) and (iv) are the same as the statements obtained by Yang and So [20].

Now, we consider (2.4) under condition (2.2) and assume that $r, \tau, \beta$ and $m>1$ are positive constants. Suppose that $\frac{\beta}{r}>1$. Take $y_{0}=\left(\frac{1}{m-1}\right)^{\frac{1}{m}}$ and $\left(\frac{\beta-r}{r}\right)^{\frac{1}{m}}=$ $K$. Using the same argument as in above, we will get the following conclusions:

(a1) If $1<\frac{\beta}{r}<\frac{m}{m-1}$ and $g_{k}(x)$ satisfies (H3) and $M_{0} \leq\left(y_{0}-\frac{f\left(y_{0}\right)}{\delta}\right)\left(1-e^{-\delta \gamma}\right)$, then every solution $u(t, x)$ of (2.4) under condition (2.2) converges to $K$ (uniformly in $\mathrm{x}$ )as $t \rightarrow \infty$.

(a2) If the condition $\frac{\beta}{r}>\frac{m}{m-1}, g_{k}^{\prime}(K)>-1$ and $\frac{r[\beta(m-1)-m r]}{\beta} e^{r \tau} \liminf _{t \rightarrow \infty} \int_{t-\tau}^{t} \prod_{s-\tau \leq t_{k}<s}\left(1+g_{k}^{\prime}(K)\right)^{-1} d s>\frac{1}{e}$ hold and $g_{k}(x)$ satisfies (H3) and then every solution $u(t, x)$ of (2.4) under condition (2.2) oscillates about positive equilibrium $K$ in $D$.

If $g_{k}(x) \equiv 0$ for any $k \in I_{\infty}$ in (2.3), then we have the following results.

(a3) If $1<\frac{\beta}{r} \leq \frac{m}{m-1}$ holds, then every solution $u(t, x)$ of (2.4) under condition (2.2) converges to $K$ (uniformly in $\mathrm{x}$ ) as $t \rightarrow \infty$.

(a4) If $\frac{p}{\delta}>\frac{m}{m-1}$ and $\frac{r \tau[\beta(m-1)-m r]}{\beta} e^{r \tau}>\frac{1}{e}$ hold, then every solution $u(t, x)$ of (2.4) under condition (2.2) oscillates about positive equilibrium $K$ in $D$.

(a5) If $\frac{\beta}{r}>1$, then every non-oscillatory solution $u(t, x)$ of (2.4) under condition (2.2) about positive equilibrium $K$ in $D$ converges to $K$ (uniformly in x)as $t \rightarrow \infty$.

Remark 6.2. Our results are new for the diffusive model of Hematopoiesis (2.4) with conditions (2.2). Moreover, when (2.4) and (2.2) is not effected by impulses and a spacial variable $x \in \Omega$, that is

$$
\left\{\begin{array}{l}
u^{\prime}(t)=-r u(t)+\frac{\beta u(t-\tau)}{1+u^{m}(t-\tau)}, t>0 \\
u(\theta)=\phi(\theta) \geq 0, \theta \in[-\tau, 0],
\end{array}\right.
$$

our result (a3)-(a5) are also true. In addition, in (a3), our condition $1<\frac{\beta}{r} \leq \frac{m}{m-1}$ 
is stronger than the condition $1<\frac{\beta}{r}<\frac{4 m}{(m-1)^{2}}$ for $1<m<5$ and weaker for $m \geq 5$ in Kuang[10]. In (a5), our condition $\frac{\beta}{r}>1$ is weaker than the condition $\frac{\beta}{r}>2$ in Saker[17].

Acknowledgment. The authors thank the referee for careful reading of the manuscript and useful suggestions that helped to improve the paper.

\section{References}

[1] D. D. Bainov and E. Minchev, Oscillation of the solutions of impulsive parabolic equations, J. Comput. Appl. Math., 69(1996), 207-214.

[2] C. Cui, M. Zou, A. Liu and L. Xiao, Oscillation of nonlinear impulsive parbolic differential equations with several delays, Ann. of Diff. Eqs., 21(1)(2005), 1-7.

[3] T. Ding, Asymptotic behavior of solution of some retarded differential equations, Scientia Sinica(A), 25(4) (1982), 363-370.

[4] L. H. Erbe, H. I. Freedman, X. Liu and J. H. Wu, Comparison principles for impulsive parabolic equations with applications to models of single species growth, J. Austral. Math. Soc. Ser. B32(1991), 382-400.

[5] X. Fu, L. Shiau, Oscillation criteria for impulsive parabolic boundary value problem with delay,Applied Mathematics and Computation, 153(2004), 587-599.

[6] X. Fu, X. Liu and S. Sivaloganathan, Oscillation criteria for impulsive parabolic differential equations with delay, J. Math. Anal. Appl., 268(2002), 647-664.

[7] X. Fu, B. Q. Yan and Y. S. Liu, Introduction to impulsive differential systems(in Chinese), Science Press, Beijing, 2005.

[8] W. Gao and J. Wang, Estimates of solutions of impulsive parabolic equations under Neumann boundary condition, J. Math. Anal. Appl., 283(2003), 478-490.

[9] M. Kirane and Y. V. Rogovchenko, Comparison results for systems of impulse parabolic equations with applications to population dynamics, Nonlinear Anal. TMA., 28(2)(1997), 263-276.

[10] Y. Kuang, Delay Differential Equations with Applications in Population Dynamics, Academic Press, New York, 1993.

[11] M. R. S. Kulenovic, G. Ladas and A. Meimaridou, On oscillation of nonlinear delay differential equations. Quarterly Of Applied Mathematics, XLV(1)(1987), 155-164.

[12] W. Li, On the forced oscillation of solutions for systems of impulsive parabolic differential equations with several delays, J. Comput. Appl. Math., 181(2005), 46-57.

[13] W. Li ,M. Han and F. Meng, Necessary and sufficient conditions for oscillation of impulsive parabolic differential equations with delays, Applied Mathematics Letters, 18(2005), 1149-1155. 
[14] M. C. Mackey and L. Glass, Oscillation and chaos in physiological control system, Science, 197(1977), 287-289.

[15] R. Redlinger. Exitence theorems for Semiliner parabolic Systems with Functional, Nonlinear Anal. TMA., 8(1984), 667-682.

[16] R. Redlinger, On Volterra's population equation with diffusion, SIAM J. Math. Anal., 16(1985), 135-142.

[17] S. H. Saker, Oscillation and global attractivity in hematopoiesis model with delay time, Applied Mathematics and Computation, 136(2003), 241-250.

[18] O. O. Struk and V. I. Tkachenko, On impulsive lotka-volterra systems with diffusion, Ukrainian Mathematical Journal, 54(4)(2002), 629-646.

[19] J. R. Yan and Ch. Kou, Oscillation of Solutions of Impulsive Delay Differential Equations, J. Math. Anal. Appl., 254(2001), 358-370.

[20] Y. Yang, J. W. -H. So, Dynamics for the diffusive Nicholson blowflies equation, in: Proceedings of the International Conference on Dynamical Systems and Differential Equations, held in Springfield, Missouri, USA, May 29-June 1, 1996. 